\title{
North Europe power transmission system vulnerability during extreme space weather
}

\author{
Roberta Piccinelli and Elisabeth Krausmann* \\ European Commission, Joint Research Centre, Via E. Fermi 2749, 21021 Ispra, VA, Italy
}

Received 18 November 2016 / Accepted 10 November 2017

\begin{abstract}
Space weather driven by solar activity can induce geomagnetic disturbances at the Earth's surface that can affect power transmission systems. Variations in the geomagnetic field result in geomagnetically induced currents that can enter the system through its grounding connections, saturate transformers and lead to system instability and possibly collapse. This study analyzes the impact of extreme space weather on the northern part of the European power transmission grid for different transformer designs to understand its vulnerability in case of an extreme event. The behavior of the system was analyzed in its operational mode during a severe geomagnetic storm, and mitigation measures, like line compensation, were also considered. These measures change the topology of the system, thus varying the path of geomagnetically induced currents and inducing a local imbalance in the voltage stability superimposed on the grid operational flow. Our analysis shows that the North European power transmission system is fairly robust against extreme space weather events. When considering transformers more vulnerable to geomagnetic storms, only few episodes of instability were found in correspondence with an existing voltage instability due to the underlying system load. The presence of mitigation measures limited the areas of the network in which bus voltage instabilities arise with respect to the system in which mitigation measures are absent.
\end{abstract}

\section{Introduction}

Space weather driven by solar activity can cause serious impacts on power transmission systems. Geomagnetic disturbances (GMD) triggered by solar activity can lead to variations in the geomagnetic field (Boteler, 2001). As expressed by Faraday's law of induction, perturbations of the geomagnetic field to the ground level induce a geoelectric field at the earth's surface which, in turn, causes geomagnetically induced currents (GICs) (Bernabeu, 2013) to enter through the grounding connections of the high voltage transmission grids and flow throughout the system. This can lead to power grid instabilities and even grid collapse.

The root cause of the problem is associated with power system transformers. GICs are quasi-DC currents and, when injected into the power grid, they can drive a power transformer into half-cycle saturation, increasing the reactive power consumed by the transformer, injecting even and odd harmonics into the system, and potentially generating hotspots in the structural components. These effects may present themselves alone or combined and may result in system-wide disturbances or, eventually, in blackouts (JASON, 2011; NERC, 2012). Two

\footnotetext{
*Corresponding author: el isabeth.krausmann@ec.europa.eu
}

well-known episodes of power grid collapse related to a GMD are the Hydro-Quebec blackout in March 1989 (Bolduc, 2002) and a blackout in Sweden in 2003 (Wik et al., 2008).

GIC flows are a function of several parameters which include: geomagnetic latitude, system topology (e.g. line length and orientation), line resistance, transformer type, and geoelectric field magnitude and orientation (NERC, 2012). For instance, the effects of geomagnetic storms are often confined to high magnetic latitudes. In case of extreme storms, also lower latitudes can be affected but less so than in high-latitude areas. Line length and line orientation are two fundamental topological parameters of the power network that directly influence the GIC magnitude in the system. The longer the transmission line and the closer the alignment of the line orientation and the geoelectric field, the higher will be the current induced in the network. Line resistance also influences GIC magnitude in the system: high-voltage lines, which usually have a lower resistance than low-voltage lines, allow important amounts of GIC to flow throughout the system. Transformer design is also a major determinant in understanding the vulnerability of the power grid, as some types are more susceptible to damage than others. Threephase transformers, for instance, have been found to be less vulnerable than single-phase transformers when subjected to the same levels of GIC. 

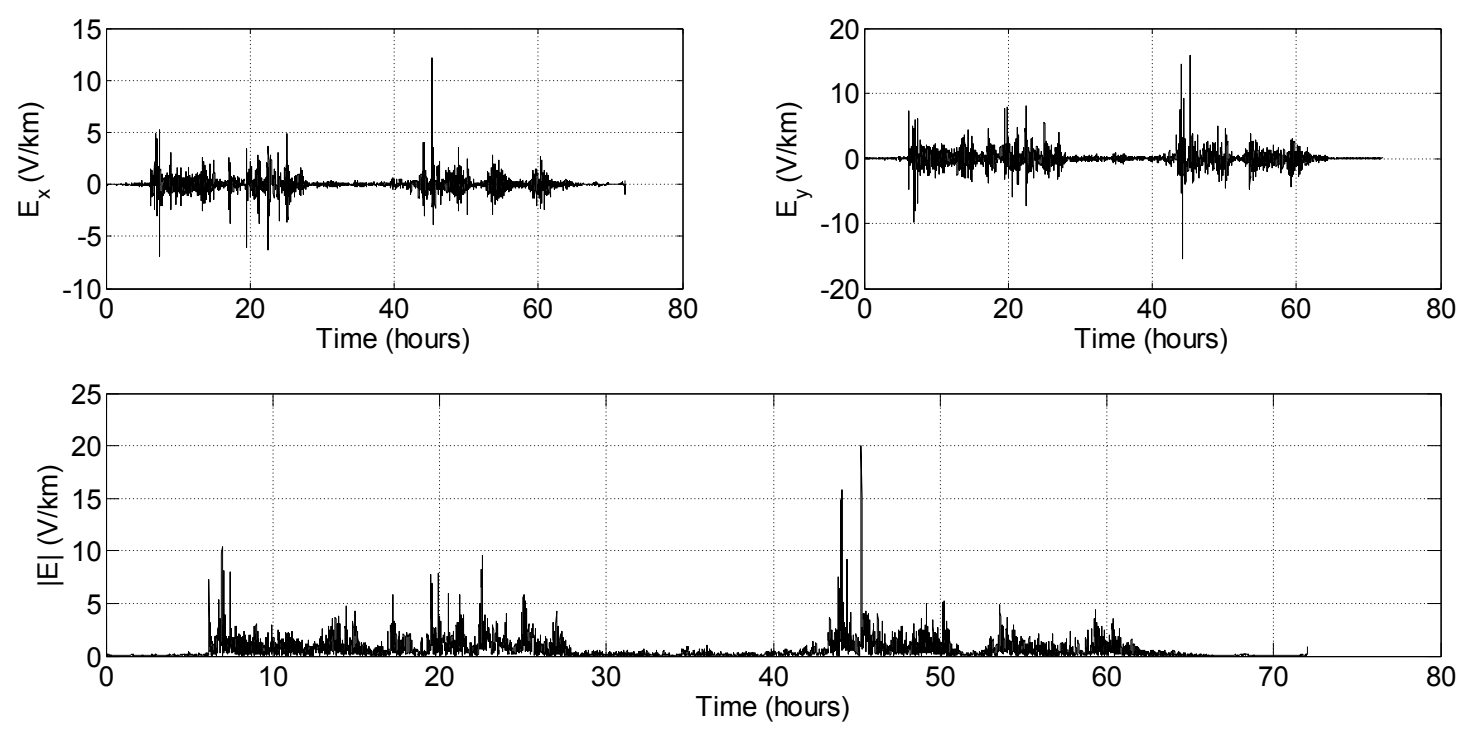

Fig. 1. Representation of geolectric field values during a 100-year GMD calculated for high latitudes and low conductivity soil. The maximum intensity of the geoelectric field is $|\boldsymbol{E}|=20 \mathrm{~km} / \mathrm{V}$. Data of the time series and details can be found in Pulkkinen et al. (2012).

The study of the effects of GIC flow in a power transmission system also needs to include an analysis of the behavior of the system following GIC injection. For instance, using first principles, Boteler (2014) proposes an efficient method for simulating the GIC produced in a power system during a major GMD. The method is based on power system analysis and Boteler presents how the methods normally used for AC modelling can be adapted to model the quasi-DC GICs. Albertson et al. (1981) carried out load-flow studies in the presence of GIC in the northern United States and Canada. Their results clearly show serious voltage drops and real and reactive power flow changes during a GMD. As a consequence, they propose GIC mitigation measures in particular for power systems that are susceptible to GIC due to their location and network topology. Bernabeu (2013) presents the methodology used by Dominion Virgina Power to model GIC. Using an extreme 100-year geoelectric field scenario as baseline, the impact of topology changes in the analyzed power grid was investigated to identify critical locations and weaknesses in the system with a view to updating operational procedures and GIC mitigation strategies. Studies using the electromagnetic transient approach for understanding the impact of a GMD were carried out by Gerin-Lajoie et al. $(2013,2014)$. They focus on transformer response resulting from the injection of GICs and propose a set of simulation case studies to illustrate the impact of GICs on voltage regulation in the presence of transformer saturation. Walling and Khan (1991) present a detailed study of the characteristics of transformer excitation currents during GMD. They show that transformer core type is an important characteristic in determining how a transformer will respond to GIC insertion while transformer-winding air-core impedance has only a moderate impact.

In this paper, we present a study of the behavior of the North European power transmission grid with different transformer configurations subjected to an extreme space weather event. Following a preliminary study (Piccinelli and Krausmann, 2015), we extend the analysis to a more detailed model of the power system, considering line compensation as an example of an existing mitigation strategy against voltage drops in the $\mathrm{AC}$ regime.

\section{Methodology}

Calculation of the GICs throughout power systems involves the evaluation of the geoelectric field and the calculation of the induced currents at nodes and lines of the system. These two tasks can be carried out separately. Additionally, the behavior of the power system following a severe geomagnetic storm needs to be modeled. This entails the evaluation of the power system in its operational mode in the $\mathrm{AC}$ regime during an extreme space weather event.

Power transmission grids are large-scale systems in which each transformer experiences different levels of GIC during space weather events. In order to describe the impact of a GMD on the system, we propose a hybrid approach. For describing the backbone structure of the grid we use the network-theory approach, which delineates the topology of the grid as a graph in which nodes represent stations of the system linked by edges representing the transmission lines (Pagani and Aiello, 2013). Physical properties and flow models are added in the modeling of the network to allow a detailed and technical representation of the grid. Finally, functional relationships, linking GICs to the reactive power in the system, model the behavior and the stress caused by GICs on the system components (Dong et al., 2001). This is further discussed in Section 2.4.

\subsection{Extreme GMD scenario}

In studying the impact of extreme space weather on the power grid, we adopt the GMD scenario proposed by Pulkkinen et al. (2012). The Pulkkinen et al. (2012) model is a benchmark of extreme events which are defined as the maximum 100-year amplitude of the 10-second resolution horizontal geoelectric field obtained as linear extrapolation 


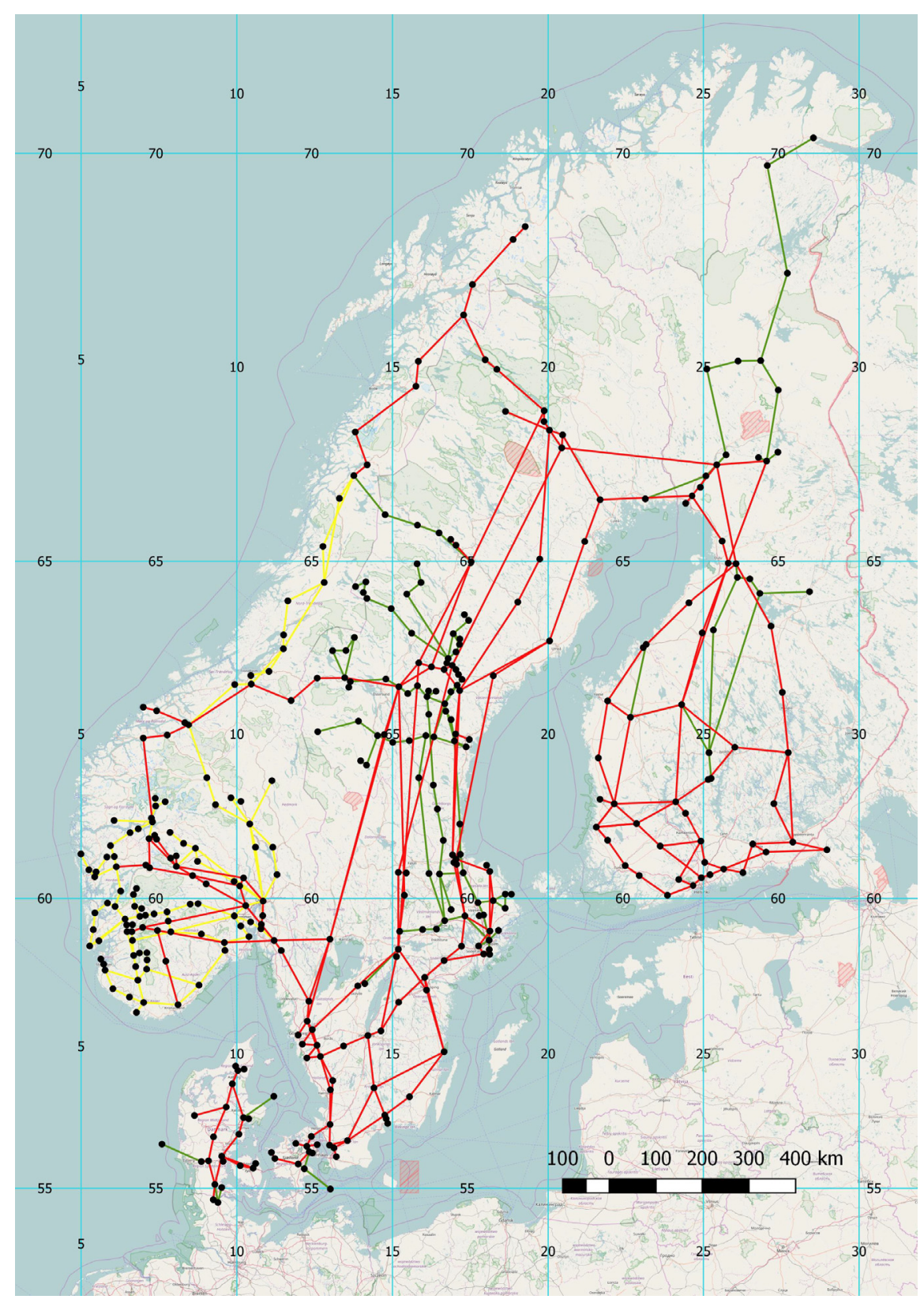

Fig. 2. The northern portion of the European high voltage power grid includes $400 \mathrm{kV}$ (red), 300-330 kV (yellow) and 200-220 kV (green) transmission lines (schematic adapted from $\left.\operatorname{ENTSOE}^{1}(2015)\right)$. The black dots are substations.

of 13 years of geomagnetic-field recording. Key parameters for the evaluation of the modelled geoelectric field are: (1) ground conductivity, (2) geomagnetic latitude, and (3) temporal and spatial scales of extreme geoelectric fields. Pulkkinen et al. (2012) generated four extreme scenarios as a function of two extreme ground conductivities (low and high conductivity), and the location of the power system with respect to a defined geomagnetic latitude threshold at around $50-55^{\circ}$ (high latitudes above the threshold and low latitudes below the threshold). High latitude scenarios (high latitude/low conductivity and high latitude/high conductivity) show a larger magnitude, by a factor of ten, of the associated geoelectric field $|\boldsymbol{E}|$ with respect to the low latitude scenarios. For the same latitude, the geoelectric-field magnitude $|\boldsymbol{E}|$ changes by a factor of five when passing from low to high conductivity. Therefore, latitude plays a predominant role compared to conductivity for the magnitude of the geoelectric field. Since the North European power transmission system is located above the geomagnetic threshold, we will apply the scenario from Pulkkinen et al. (2012) which refers to high latitudes (above the aforementioned geomagnetic threshold) and resistive soil, and which constitutes a worst-case scenario. For the purpose of this study we will refer to this scenario as Scenario A. Figure 1 summarizes the intensity of the geoelectric field during a severe geomagnetic storm with peak amplitude of $|\boldsymbol{E}|=20 \mathrm{~V} / \mathrm{km}$. Data of the time series and details can be found in Pulkkinen et al. (2012). 
The model proposed by Pulkkinen et al. (2012) is derived with reference to Canadian soil conductivity models. Usually, ground models derived for a specific geographical region cannot simply be applied to geomagnetic observations of different geographical areas. However, Pulkkinen et al. (2012) state that in the absence of nearby conductivity anomalies, the same magnetospheric current will to a good approximation generate similar total magnetic variations even if ground conductivity structures are different.

In our case, Scenario A was applied to studying the impact of a GMD on the North European region, since the highest geoelectric field intensity ever recorded in Sweden was $|\boldsymbol{E}|=20 \mathrm{~km} / \mathrm{V}$ in the $1920 \mathrm{~s}$ (Pirjola, 1983).

\subsection{GIC assessment}

For the assessment of GICs we follow the procedure proposed by Lehtinen and Pirjola (1985). A power grid is a discrete grounded system with $n$ nodes, also called earthing points. GICs, $\mathbf{I}^{e}$, are then calculated according to:

$$
\mathbf{I}^{e}=\left(\mathbf{U}+\mathbf{Y}^{n} \mathbf{Z}^{e}\right)^{-1} \mathbf{J}^{e}
$$

where $\mathbf{U}$ is the $n \times n$ unit matrix, and $\mathbf{Y}^{n}$ and $\mathbf{Z}^{e}$ are the $n \times n$ network admittance matrix and $n \times n$ earthing impedance matrix, respectively. The off-diagonal element of the network admittance matrix $\mathbf{Y}^{n}$ is defined as the inverse of the conductor line resistance between nodes $i$ and $j, R_{i j}{ }^{n}$ :

$$
Y_{i j}^{n}=-\frac{1}{R_{i j}^{n}}(i \neq j)
$$

and the diagonal element is defined as the sum of the inverse of the line resistances between node $i$ and the adjacent nodes $j$

$$
Y_{i i}^{n}=\sum_{j \neq i} \frac{1}{R_{i j}^{n}}
$$

The elements of the $n \times 1$ column matrix of the "perfectearthing" current $\mathbf{J}^{n}$ are defined by:

$$
\begin{gathered}
J_{i}^{e}=\sum_{j \neq i} J_{i j}^{n} \\
J_{i j}^{n}=\frac{V_{i j}}{R_{i j}^{n}} \\
V_{i j}=\int_{S_{j i}} \overrightarrow{\mathbf{E}} \cdot d \overrightarrow{\mathbf{s}}
\end{gathered}
$$

The conductor line from node $i$ to node $j$ is denoted as $s_{j i}$ and $\boldsymbol{E}$ is the geoelectric field. Thus $V_{i j}$ is the voltage affecting the line $s_{j i}$.

\subsection{The power transmission system}

The network model base for our study was the map of the interconnected network of Northern Europe provided by the
Table 1. Nodes numbering and location.

\begin{tabular}{ll}
\hline Nodes & Country \\
\hline From node 1 to node 64 & Finland \\
From node 65 to node 215 & Sweden \\
From node 216 to node 341 & Norway \\
From node 342 to node 347 & East Denmark \\
\hline
\end{tabular}

European Network of Transmission System Operators for Electricity (ENTSOE $\left.{ }^{1}(2015)\right)$. We considered the northern synchronous area of the grid, which comprises Finland, Sweden, Norway and the east of Denmark. Since high voltage lines are more susceptible to GMD, the system includes only line voltage levels above $200 \mathrm{kV}$ (Fig. 2). Line orientations and lengths are important parameters. We therefore built our model by mapping each transmission line connecting two substations as piecewise lines instead of one straight line to account for local line orientation effects.

The following information and assumptions were used for the power system:

- the network is composed of 347 nodes and 469 lines. Table 1 summarizes the numbering of nodes and the countries they belong to;

- we included all substations and lines with voltages equal to or above $200 \mathrm{kV}$ : $200-220 \mathrm{kV}, 300-330 \mathrm{kV}$ and $400 \mathrm{kV}$. High voltage direct current connections are not taken into account;

- when dealing with earthing impedance, it should be considered that individual stations may influence GIC values at the stations closest to them. If power grids are considered as a whole, it was shown by Pirjola (2008a) that when all stations are far enough from each other, the mutual earthing impedance can be ignored. Since in our model of the transmission power system the shortest distances of lines are as high as $20 \mathrm{~km}$, we can omit the mutual earthing impedance;

- values assumed for the line resistances per unit length were $0.008 \Omega / \mathrm{km}$ for $400 \mathrm{kV}$ lines (Elovaara, 2007; Pirjola, $2008 \mathrm{~b}$ ), and $0.022 \Omega / \mathrm{km}$ for $220 \mathrm{kV}$ and $300-330 \mathrm{kV}$ lines (Norway) (Viljanen et al., 2012).

Data on power grids are usually not publicly available due to security reasons. Consequently, data for our model of the North European power transmission grid were inferred from open source information:

- The system has a total installed capacity of $43000 \mathrm{MW}$ (Enipedia $^{2}$, 2016; ENTSOE ${ }^{1}, 2015$ );

- To model the system in a situation of highest load request, the total load considered consumption during winter time.

\footnotetext{
${ }^{1}$ ENTSOE, European Network of Transmission System Operators for Electricity 2015, https://www.entsoe.eu/map

${ }^{2}$ Enipedia, 2016, http://enipedia.tudelft.nl
} 
A load profile with a peak value of $35000 \mathrm{MW}$ was considered $\left(\mathrm{SVK}^{3}, 2016\right.$; Fingrid ${ }^{4}, 2016$; Statkraft $^{5}, 2016$; Energinet $\left.^{6}, 2016\right)$ and assigned to load busses of the network proportionally to the population density;

- The transmission grid has some series compensated lines (Fig. 3): by insertion of capacitors, GICs cannot flow across compensated lines and thus, their propagation through the system is limited. Line compensation was taken into account for the eight longest lines of the Swedish grid, traversing $62^{\circ}$ of geographic latitude, and for the two lines connecting Finland and Sweden (Arnborg, personal communication; Elovaara, 2005). In the absence of public information on the number of transformers per substation, we reasonably assumed for the purpose of this study that each node has a transformer. We also assumed that all transformers of the network are core type, present the same characteristics and that their earthing resistance is equal to $0.28 \Omega$ (Pirjola, 2008a).

\subsection{Transformer behavior}

High voltage transformers are particularly sensitive to GICs. Quasi-DC current flows through the transformer windings, causing transformer saturation and increasing leakage fluxes in the transformers (Dong et al., 2001). Consequences of transformer saturation are an increase in the reactive power demand in the system, which may cause a reactive power imbalance and voltage instability for the entire system (ES), and the generation of harmonic currents that can induce protection relays to actuate incorrectly, thereby triggering unnecessary tripping and leading to system collapse. Increased leakage flux can cause hot spots in the metallic parts of the transformer and deterioration of the organic insulation material. In the following, we concentrate on transformer saturation and its impact on the voltage stability of the system. The transformers are assumed to saturate instantaneously which is a conservative assumption. The effect of GIC on transformer insulation degradation is not considered because it requires data that are not easily available.

Several studies assume a linear transformer behavior during GIC saturation (Dong, 2002; Overbye et al., 2012). The increase in reactive power consumption due to saturation caused by GICs is proportional to a coefficient $K 1$ that depends on the characteristics of the transformer core design (Dong, 2002). Table 2 gives an overview of the coefficient values for different types of transformer design.

The North European power grid has only core type transformers (Arnborg, personal communication), so we will consider only the three-phase, three-legged and three-phase, five-legged transformer cases in Table 2. The increase of reactive power $\mathbf{Q}$ drawn by the transformer is estimated using the following equation (Dong, 2002):

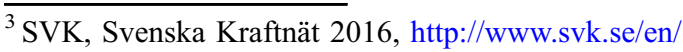

${ }^{4}$ Fingrid 2016, http://www.fingrid.fi/en/

${ }^{5}$ Statkraft 2016, http://www.statkraft.com/

${ }^{6}$ Energinet 2016, http://www.energinet.dk/EN/
}

Table 2. $K$ coefficients for different types of transformer core design (Dong, 2002).

\begin{tabular}{ll}
\hline$K 1(\mathrm{Mvar} / \mathrm{A})^{*}$ & Transformer core design \\
\hline 1.18 & Single phase \\
0.33 & Three-phase shell form \\
0.29 & Three-phase, three-legged, core form \\
0.66 & Three-phase, five-legged, core form
\end{tabular}

${ }^{*}$ Volt-ampere reactive (var) is a unit by which reactive power is expressed in an AC electric power system.

$$
\mathbf{Q}=K 1 \cdot \mathbf{I}^{e}+\mathbf{Q}_{0}
$$

where $\mathbf{I}^{e}$ is the $n \times 1$ column vector of the GIC currents calculated using equation ( 1 ) and $\mathbf{Q}_{\mathbf{0}}$ is the $n \times 1$ column vector of reactive power consumption given from the normal exciting current at nodes.

\section{Results}

Given a fixed geophysical scenario, GIC flow is a function of the system topology and is determined by line lengths, line orientation and degree of connection. Traditionally, power lines are represented as straight lines joining two substations. This assumption is correct, to a first approximation, especially when the geoelectric field $|\boldsymbol{E}|$ is assumed to be spatially uniform across the power network. The voltage that drives the GICs throughout the system is calculated as the integral of the geoelectric field along the transmission lines (Eq. (6)), and the integration of a spatially uniform field does not depend on the path chosen. However, this approximation ignores that line paths between two nodes locally vary their orientation with respect to the direction of the straight line connecting the same nodes which results in an increase of the considered line lengths. For example, if we consider the $400 \mathrm{kV}$ part of the Scandinavian power grid (Fig. 2, red lines) as being composed of straight lines between nodes, the total line length is $17716 \mathrm{~km}$, a value reduced by $12.3 \%$ if compared to the $20217 \mathrm{~km}$ calculated considering piecewise lines. Consequently, in the straight line approximation, resistance values of lines are lower. This does not affect the calculation of voltages but changes the values of the admittance matrix and hence of GICs. In this study we considered piecewise lines for a more realistic representation of the grid topology.

The North European trfansmission network features long transmission lines mainly oriented in the north-south direction, as can be seen in Figure 2. Transmission lines have an average length of $64.35 \mathrm{~km}$, except for eight lines located in Sweden which are the longest of the system with a length greater than $280 \mathrm{~km}$. Long lines are needed since large Swedish power plants are located far from the substations they need to supply. We extended a previous study (Piccinelli and Krausmann, 2015) by considering the detailed transmission power grid proposed by ENTSOE $^{1}(2015)$ as a reference. GICs at nodes and lines are represented in Figures 4 and 5, respectively (blue bars). Since we are interested in extreme space weather, GIC values are calculated for the electric field peak intensity $|\boldsymbol{E}|=20 \mathrm{~V} / \mathrm{km}$. 


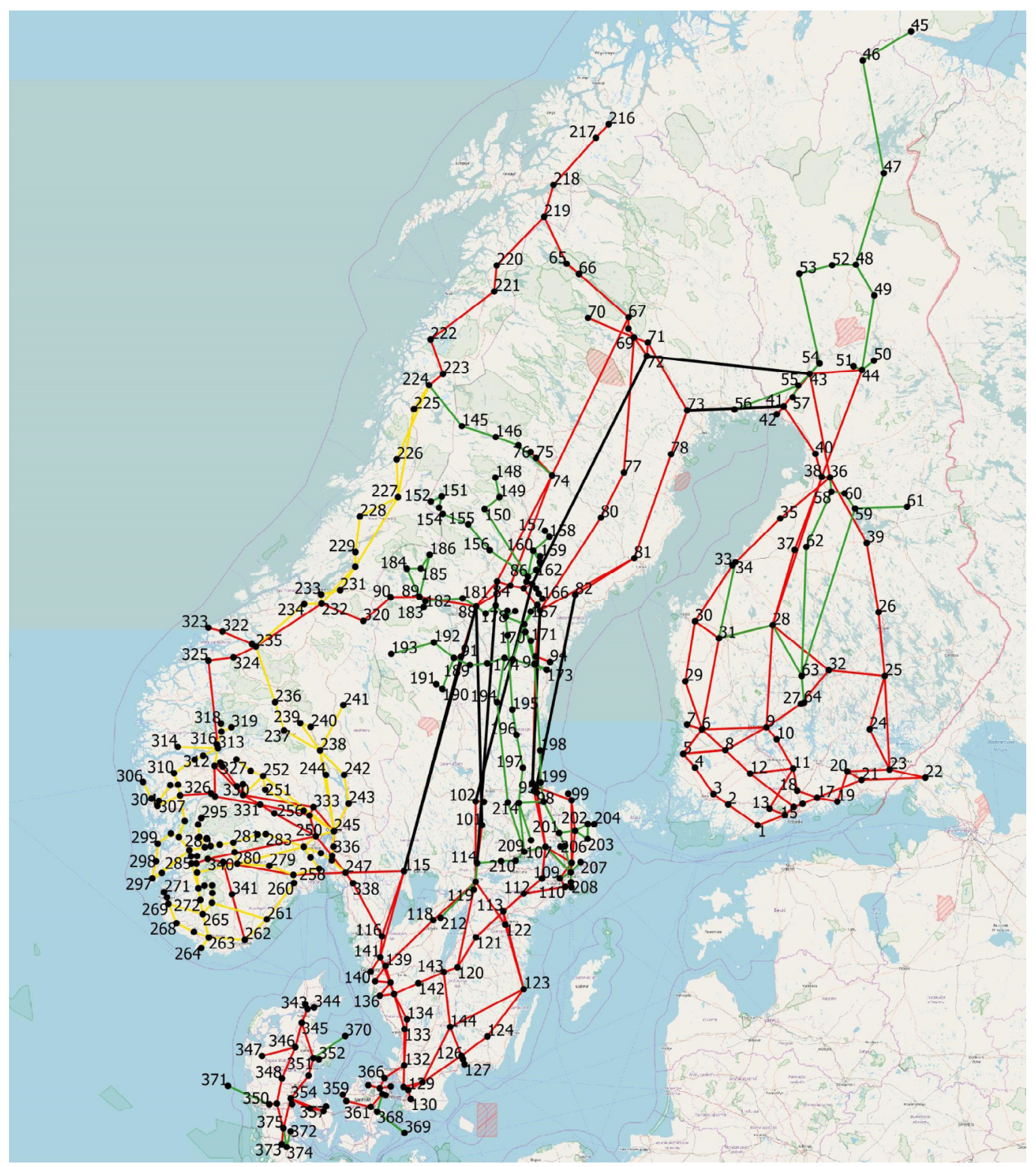

Fig. 3. The northern portion of the European high voltage power grid with node numbering and location, and indication of the compensated lines (black). Two compensated lines connect Finland to Sweden, eight compensated lines are located in the central part of Sweden.

Observations show that although rare, GIC amplitudes of several hundreds of Amperes and geoelectric field magnitudes of the order of $10 \mathrm{~V} / \mathrm{km}$ are possible (Pulkkinen et al., 2008). The largest GIC measured in the Finnish power system was as high as $201 \mathrm{~A}$ (on March 24, 1991) (Pirjola et al., 2003). Probably the largest measured GIC ever reported is $320 \mathrm{~A}$ in the Swedish power grid during the geomagnetic storm in April 2000 (Erinmez et al., 2002). Our GIC calculations are consistent with these values.In general, long lines are compensated by means of capacitors in order to increase power transfer capability as well as improving voltage stability. From a GIC point of view, compensation separates the network into smaller isolated networks because direct current cannot flow through compensated lines. This effectively comes down to removing lines from the network. GIC intensities in smaller networks may dramatically change at nodes and through lines in the proximity of the removed links, as can be seen in Figures 4 and 5 (red bars), respectively.

The North European power system has capacitors on the two $400 \mathrm{kV}$ lines connecting Sweden and Finland (Elovaara, 2005). Together with the compensation on the eight long lines crossing Sweden, they split the system into two separated subsystems: Finland and a macro region consisting of Sweden, Norway and East Denmark. Removing the compensated lines, Figure 4 shows that the increase of the difference in GIC intensity at nodes does not directly involve nodes at the ends of the removed links (i.e., nodes 41 and 54 in Fig. 3), but mainly occurs at nodes connected only with one link to the system (socalled dangling bonds) (i.e., nodes 51 and nodes 64). These nodes are mainly located in the northern part of Finland (Fig. 3). The effect of compensation on GIC at nodes is a local increase of GIC values. 

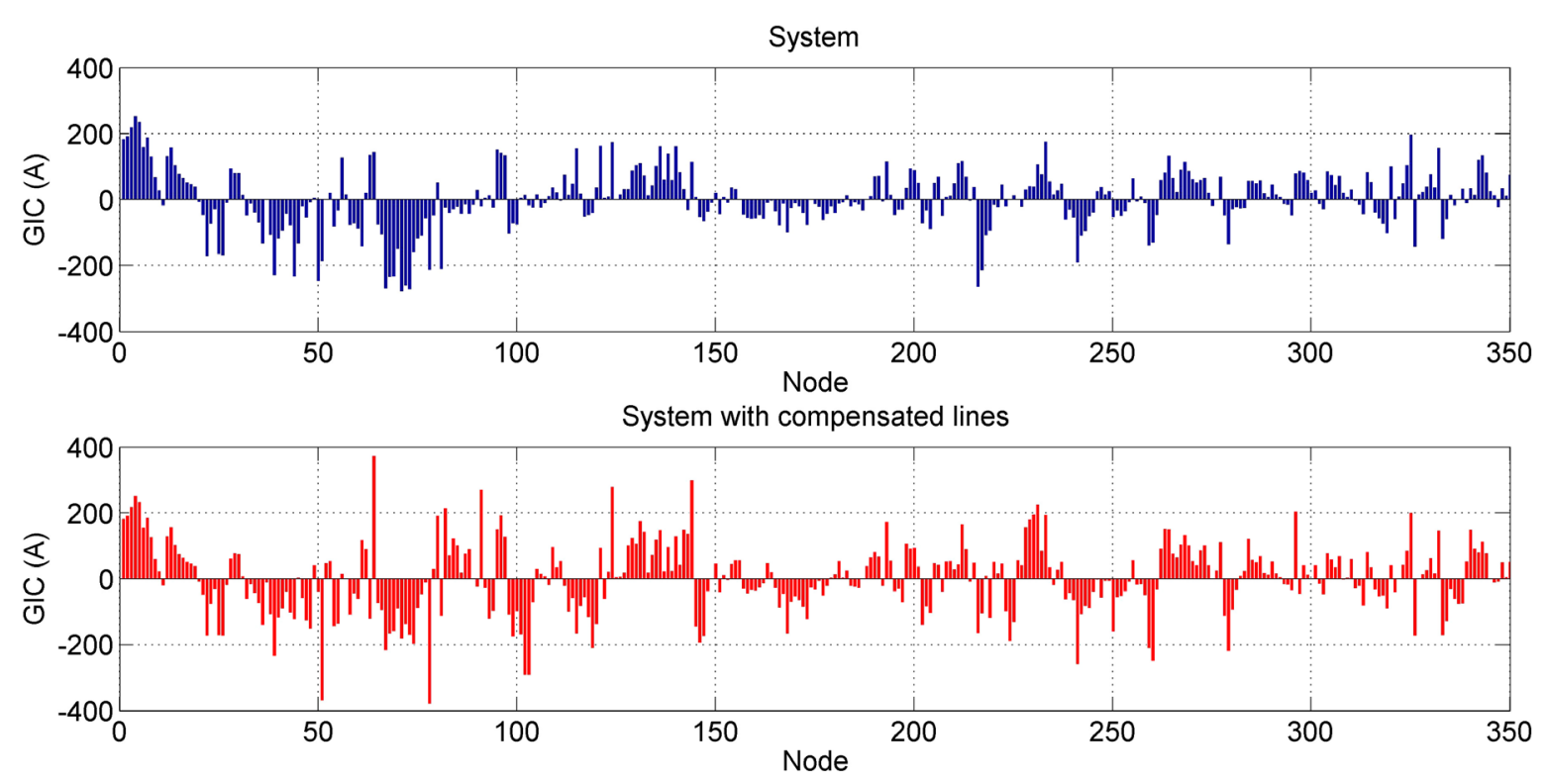

Fig. 4. Representation of GIC values at nodes when $|\mathbf{E}|=20 \mathrm{~V} / \mathrm{km}$. The blue bars represent GICs calculated when the longest lines are not compensated. The red bars represent GIC values for the compensated system. Positive/negative values denote GICs entering/leaving the system.
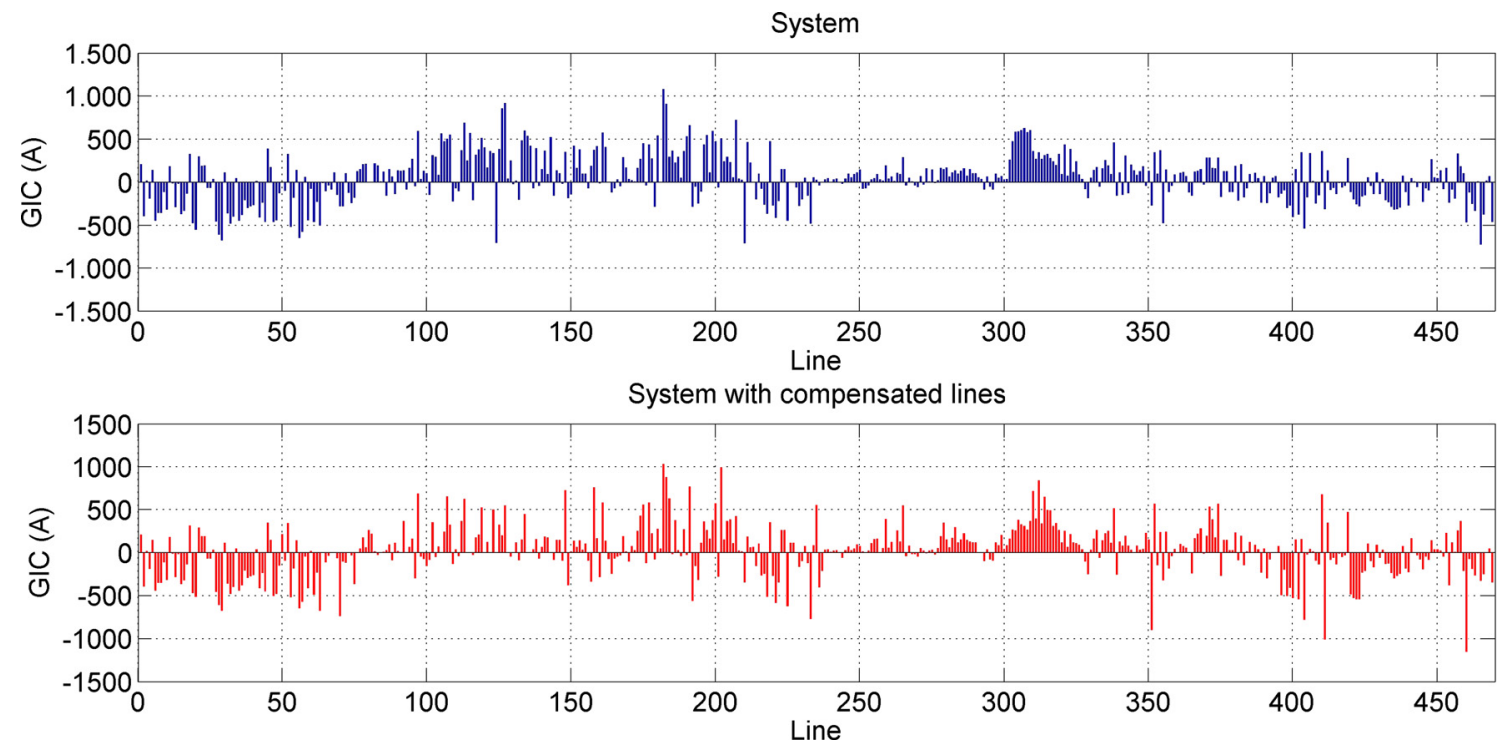

Fig. 5. Representation of GIC flowing through lines when $|\mathbf{E}|=20 \mathrm{~V} / \mathrm{km}$. The blue bars show GICs without line compensation while the red bars represent the GIC for the compensated system. GIC signs indicate the direction of the flow in the line.

Figure 5 shows how GIC flowing through lines is a process that is not local but involves the entire topology of the network. GIC effects spread through the entire network, including nodes that are far from the removed lines. Transmission lines, especially the longest ones, undergo a high increase in GIC flow. The effect of compensation is delocalized when considering GICs flowing through lines. Figure 5 shows that compensation reduces the flow in the involved lines but does not prevent high GIC flows in the neighboring lines and also throughout the system.

We extend the analysis to the entire 72-hour duration of the simulated GMD. Since GICs of both signs contribute to the saturation of transformers, we consider the absolute values of GICs flowing through each node or line and we sum over the entire time interval. We then distinguish between the sum of the absolute values of GICs calculated considering the compensation of lines which cause the system to separate into two subsystems (SS) and the absolute values of GICs for the ES with no compensation (Bernabeu, 2013):

$$
\Delta \mathrm{GIC}(t)=\left[\sum a b s\left(\operatorname{GIC}_{\mathrm{SS}}(t)\right)-\sum a b s\left(\operatorname{GIC}_{\mathrm{ES}}(t)\right)\right]
$$

The difference is shown in Figures 6 and 7 for GIC flow through nodes and lines, respectively. 


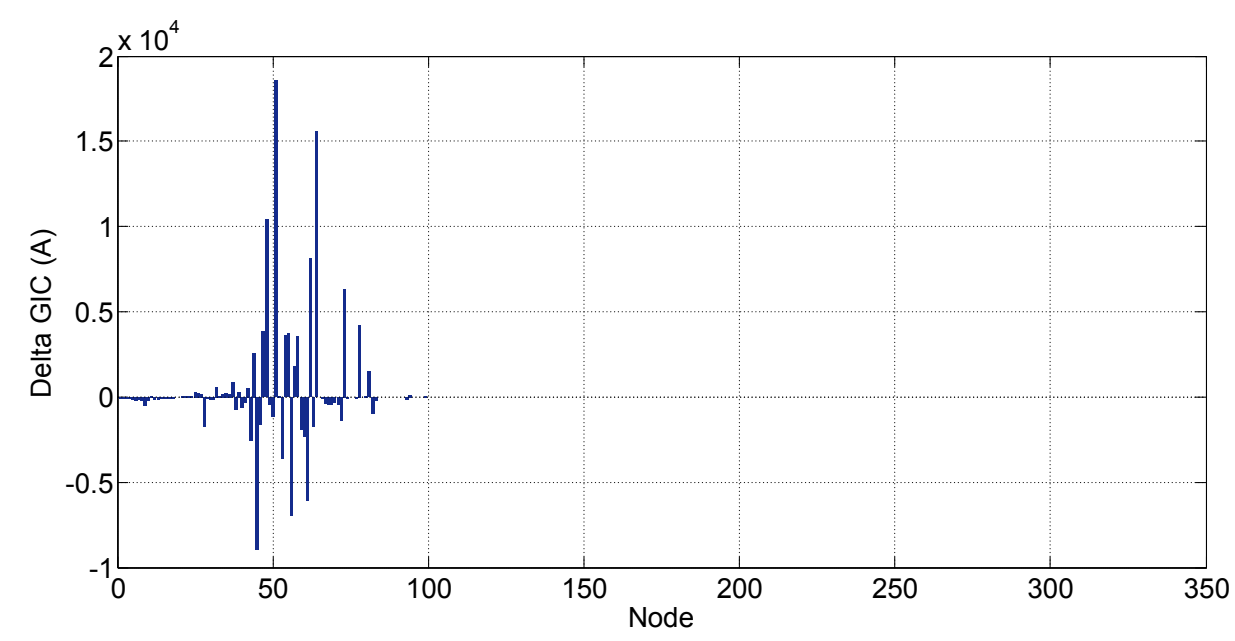

Fig. 6. Difference of the total GIC flow at nodes of the system for the entire duration of the GMD when the system is compensated (isolated) and when it is topologically connected.

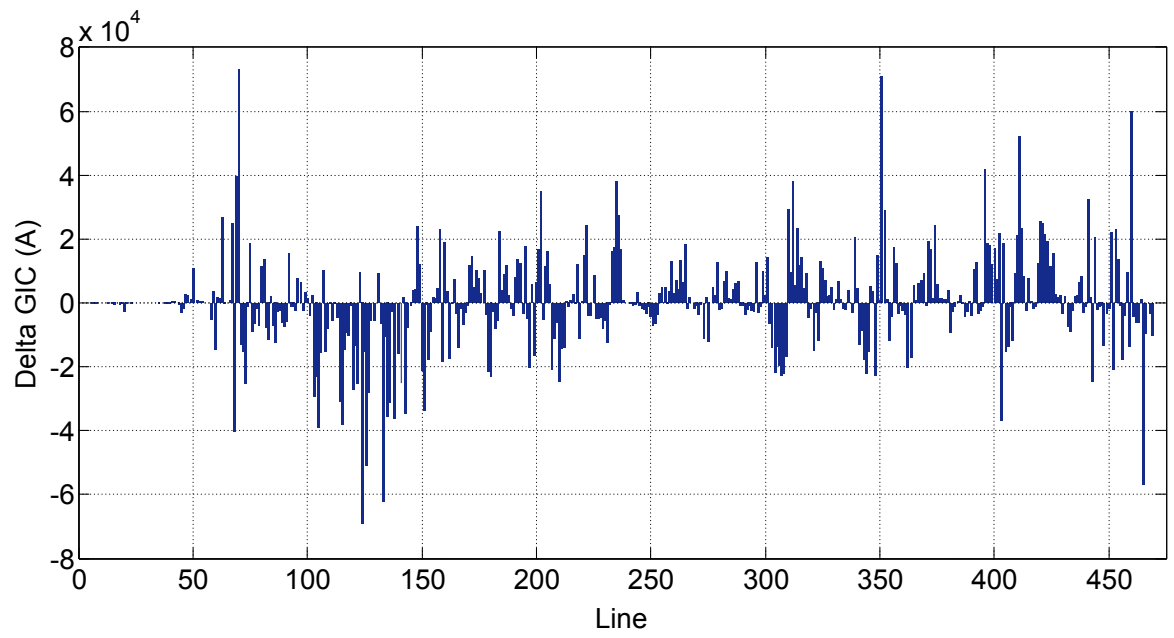

Fig. 7. Difference of the total GIC flow through the lines of the system for the entire duration of the GMD when the system is compensated and when it is topologically connected.

It should be noted that the portion of the grid that shows big differences in GIC values corresponds to nodes whose identification number is around 50 (Fig. 6). These nodes are located mainly in Finland and are represented in Figure 8: the dimension and colors of the circles around them give the difference of the total GIC flow ( $\Delta$ GIC). The red circles identify the nodes with the highest level of $\Delta \mathrm{GIC}$. It can be seen that the nodes with the highest level of difference of the total GIC flow do not coincide with the end nodes of the removed links. Indeed, the biggest differences are seen at nodes near the removed links, i.e., nodes 48 and 50, and at peripheral nodes of the Finnish system, i.e., nodes $45,56,61$ and 64. The separation of the network into two subnetworks has the effect of increasing GIC intensities at nodes of the Finnish grid with a low degree of connection or in the presence of dangling bonds, like node 64 or 45 . For the nodes of the remaining network system (Sweden, Norway and East Denmark), the effect is confined to nodes in an area close to that of the line removal, i.e. nodes 73,78 , 81 and 82.

For the subsystem composed of Sweden, with compensated lines, Norway and East Denmark, the effects of the compensation spread through the ES. The diminished number of lines results in a lower path of integration for the geoelectric field and causes GICs to diffuse throughout the ES (Fig. 7).

When a GMD induces currents in a system, the primary effect of GICs is saturation of transformers and an increase in reactive power consumption for the system. We considered transformers with either five or three limbs. In three-limb transformers, the change in flux due to GIC is smaller than for five-limb transformers (Girgis et al., 2012). Our simulations show no voltage instabilities for the system when all transformers are three-limbed. Few cases of voltage instabilities were found (Tab. 3) when the system has only five-limb transformers (Fig. 9).

Figure 9 shows bus voltage values during the episodes of grid collapse. Voltage instabilities occur when voltage values 


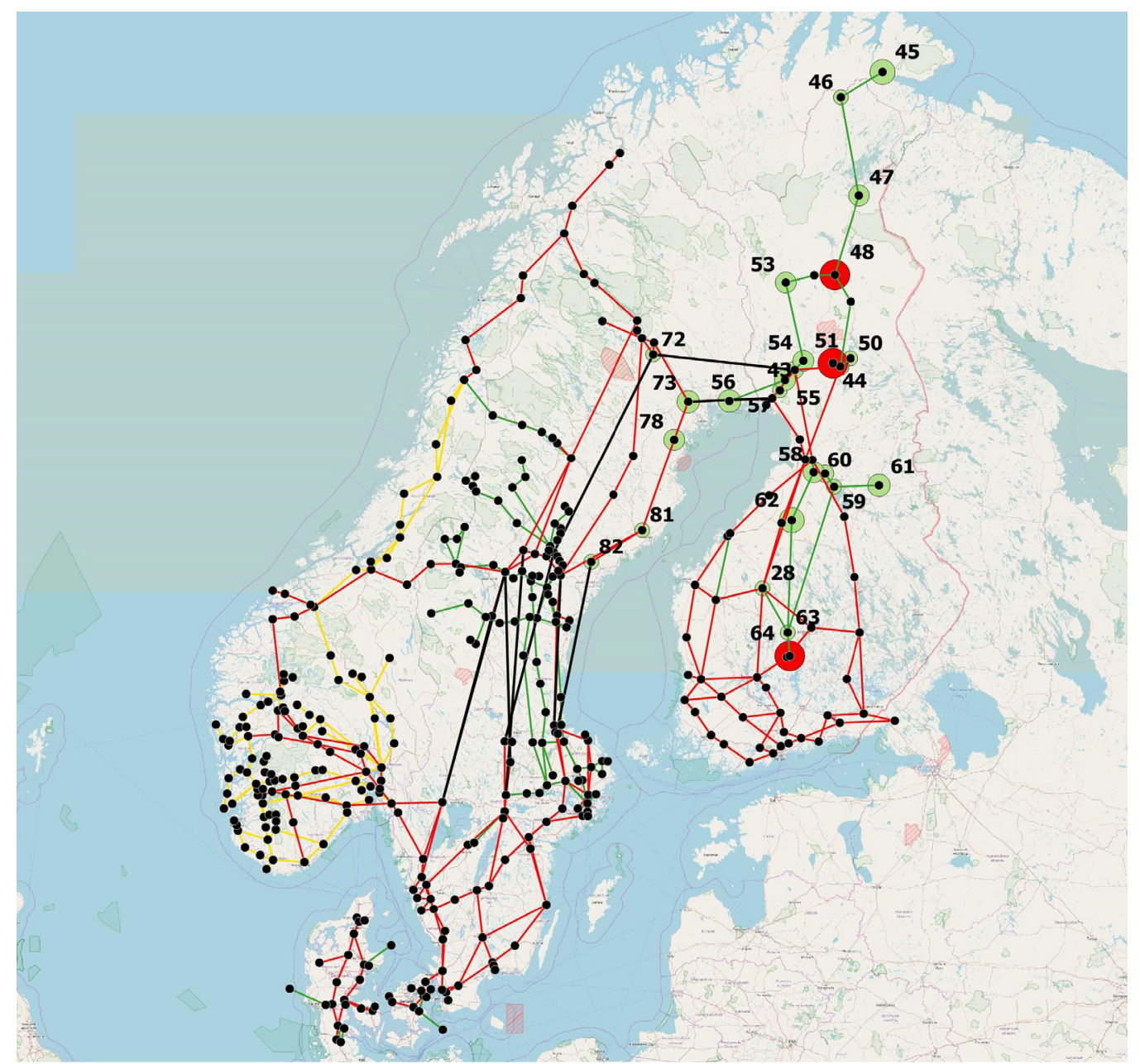

Fig. 8. Difference of the total GIC flow at nodes ( $\Delta$ GIC) of the system (green and red circles) for the entire duration of the GMD. Red circles shows the nodes with highest values in $\Delta \mathrm{GIC}$. The eight compensated lines are added in black.

Table 3. Magnitudes of $|\boldsymbol{E}|$ and time during the GMD (Fig. 1) causing the grid collapse.

\begin{tabular}{lcl}
\hline & $|\boldsymbol{E}|(\mathrm{V} / \mathrm{km})$ & Time $(\mathrm{h})$ \\
\hline Case 1 & 6.0 & 19 \\
Case 2 & 6.9 & 19.5 \\
Case 3 & 9.5 & 22.5 \\
Case 4 & 15.8 & 44 \\
\hline
\end{tabular}

at nodes exceed the range $0.95-1.05$ p.u. In our simulations, these instabilities appear to be mainly concentrated in a limited part of the network. The involved buses are located in the northern part of Finland and between Finland and Sweden (Figs. 3 and 8) and involve mainly the nodes neighboring the removed link between the two countries. These same nodes already show a "critical" voltage value during operational mode in the absence of GMD (Fig. 9, base case). Comparing the behavior of the system with or without compensation during the four collapse events, it can be seen that in the absence of compensation, the system shows diffuse voltage instabilities: in cases 1 and 2, buses with the voltage exceeding the stability values are close to node 50 (Fig. 10). In cases 3 and 4 , there is an increase in the number of nodes with voltage values outside the stable range and their distribution extends to a broad portion of the grid (Fig. 10).

The reactive power consumption due to the effects of transformer saturation and harmonics superimposes an additional strain in a system that already experiences fluctuations in voltage stability due to AC power flow constraints which eventually leads to system collapse. Reactive power losses in lines during GMD events corresponding to voltage collapse events do not differ significantly from the base case, when GMD are absent (Fig. 11).

\section{Discussion and conclusion}

The purpose of this study was to provide an understanding of the vulnerability of parts of the North European power transmission grid to extreme space weather events during its operational mode. We utilized an extreme 100-year scenario as the triggering space weather event. Although extreme, the 


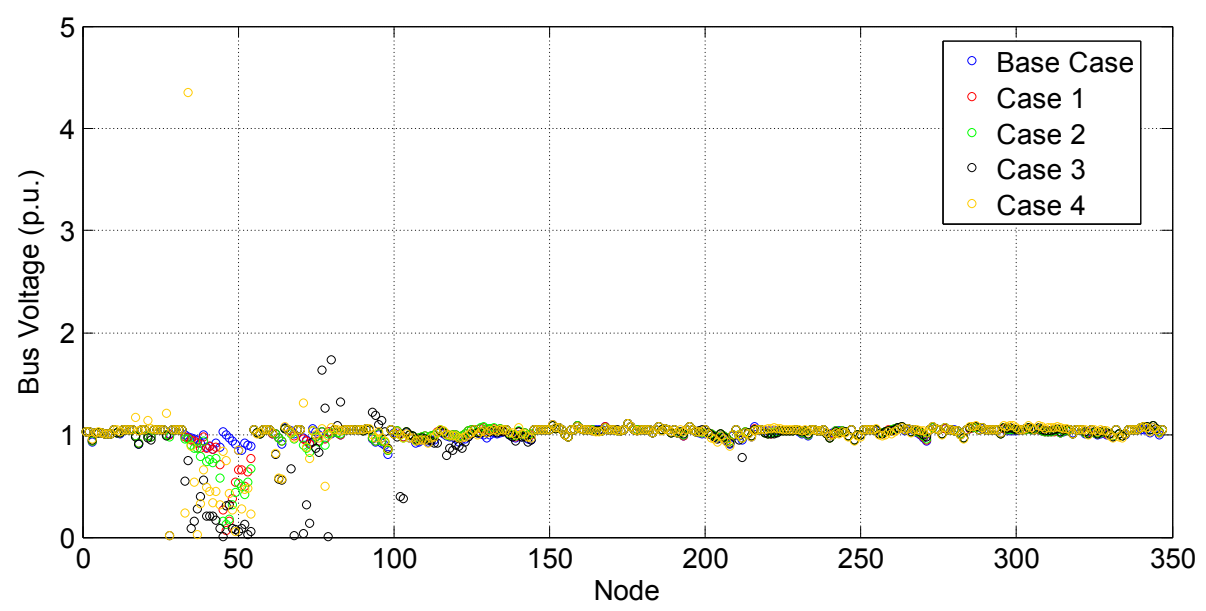

Fig. 9. Bus voltages at nodes when the system operates with no GMD (base case) and at four different time steps when the GMD causes voltage instabilities. These instabilities arise when voltage values at nodes are outside the voltage range 0.95-1.05 p.u. (per-unit). Here, the per-unit (p.u.) system expresses the bus voltage as a fraction of the nominal voltage.
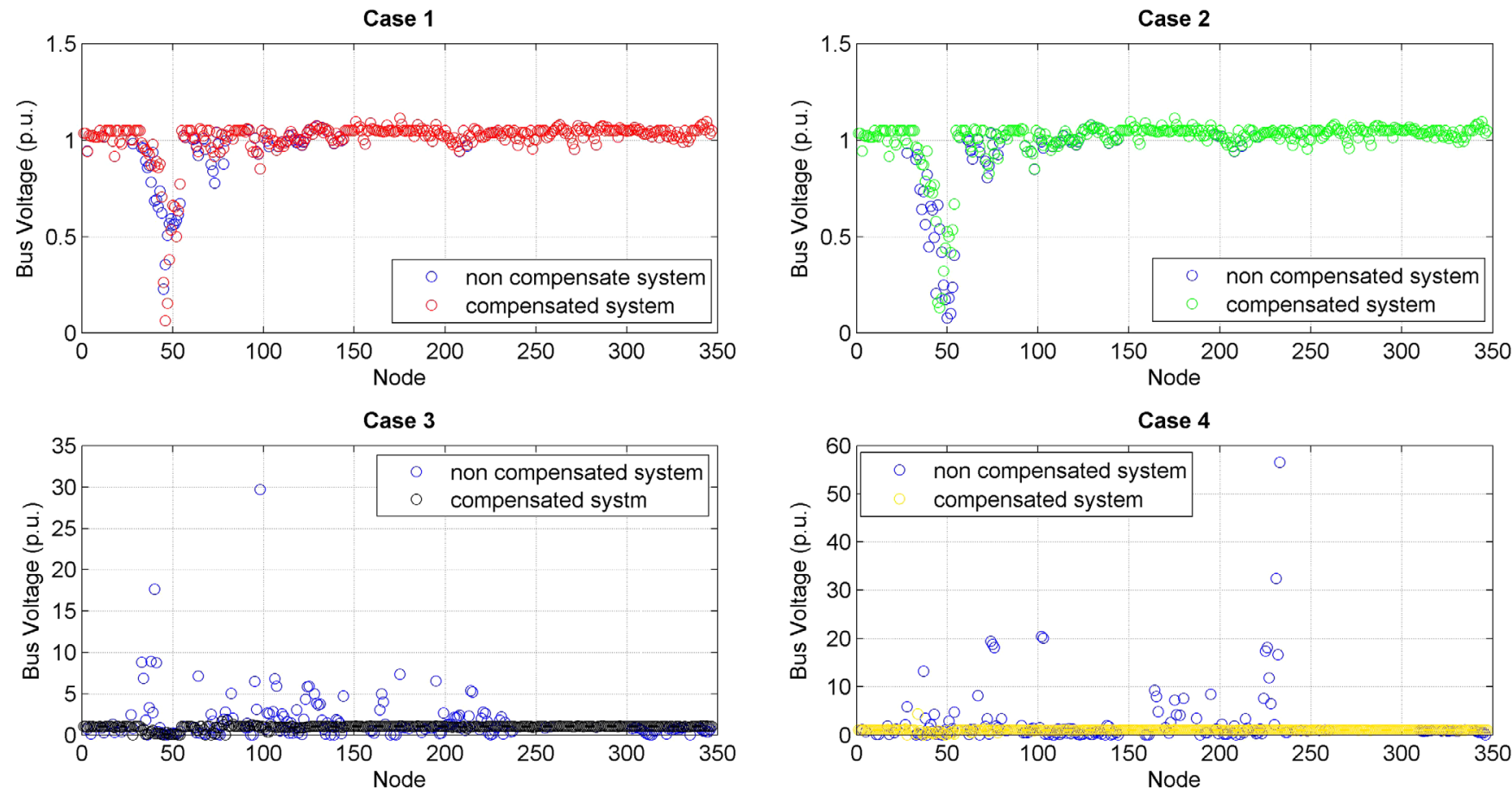

Fig. 10. Comparison of the bus voltages between the non-compensated system (blue circles) and the compensated system during the four cases of voltage instabilities identified in Table 3 .

associated geoelectric field peak magnitude of $|\boldsymbol{E}|=20 \mathrm{~V} / \mathrm{km}$ was already recorded in Sweden in the 1920s. The model of the North European power grid was built using open source data, so it necessarily is a simplified model but it can be enhanced should more detailed information be made available.

When considering GICs triggered by GMD in a power grid, the topology of the grid used for GIC and power flow calculation is usually assumed identical. Nevertheless, compensation distributed throughout the network prevents GICs from flowing along compensated lines, thus changing the topology of the network in which GICs arise. Compensated lines in Finland and Sweden divide the Nordic power grids into two separated subsystems from a GIC point of view: Finland and a sub-network composed of Sweden, Norway and East Denmark. Removing the eight longest Swedish lines does not completely disconnect the subsystems in Sweden.

GICs are spread throughout the grid by means of transmission lines. The change in the topology of the network entails a change in the GIC values calculated for lines and nodes. Removed lines cause an increase in GIC values at the nodes surrounding the end nodes of compensated lines and some nodes at the end of dangling bonds: these are the nodes 

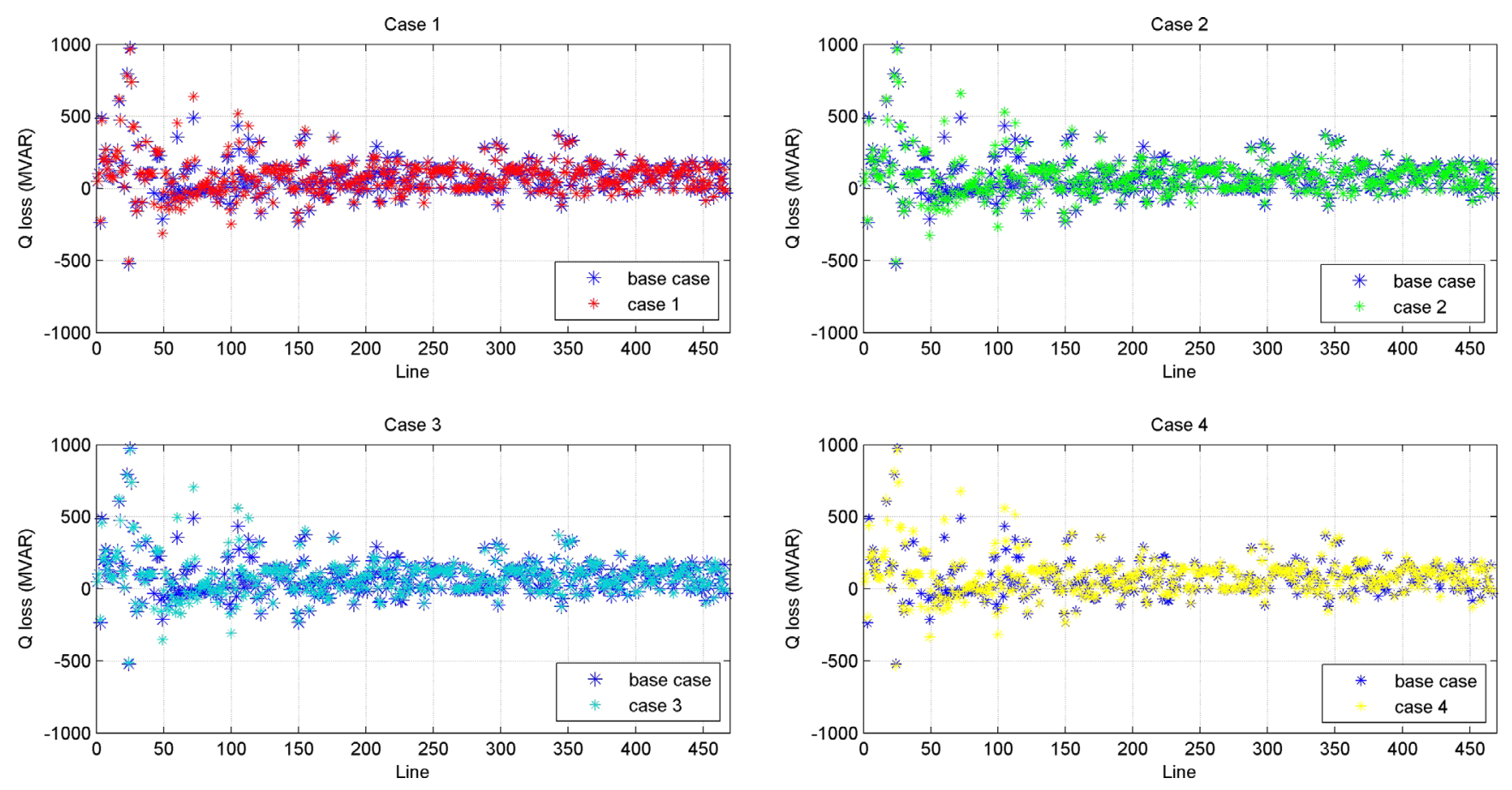

Fig. 11. Reactive losses for the lines when the systems operates with no GMD (base case, blue asterisks) and at four different time steps when the GMD causes voltage instabilities.

that experience the highest currents. In our study, the prevalent effect was on nodes located near the lines that connect Finland to Sweden and nodes in the central part of Finland. Disconnecting those lines caused a local effect, an increase in the GIC values for the neighboring nodes, and a more widespread change in the value of GICs throughout the lines of the system, since the removal of the links changes the topology of the network and therefore the paths through which GICs can spread. This characteristic is maintained during the entire seventy two-hour scenario. This means that it is an inherent characteristic of the system.

The comparison of the behavior of the considered system to the system with no compensation showed that compensation measures help to reduce the losses on lines during a GMD event, which is their primary purpose, and help to diminish the diffusion of voltage instabilities at the nodes of the system.

The topology of the network is therefore a fundamental part of the study of the vulnerability of the system since its characteristics (lines and bonds among nodes) together with the physics of the geoelectric field determine the nodes that experience the highest GICs. This preliminary study is important in view of the protection of nodes, because it can give an indication of which substations could possibly be more impacted by high currents and may therefore need more protection, thus preventing degradation and ageing in transformers components.

Nevertheless, a topological computation and analysis of GIC values at nodes and lines is not sufficient to fully understand the behavior of the power system during a GMD. GICs enter and spread throughout the system and are superimposed on the grid operational flow.

The study of the behavior of the system considering AC power flow during a space weather event has shown that the system is fairly robust to extreme space weather events. The network configuration with all transformers of the three-limb type does not suffer from voltage instability. During the geomagnetic storm simulation, no voltage collapse was found. The second configuration, with all transformers of the fivelimbed type shows few episodes of collapse, all facilitated by an existing voltage instability due to the underlying load of the system. Indeed, there is a correspondence between the part of the grid that experiences the highest GICs during the geomagnetic event and the grid portion which shows a voltage profile close to the limit of voltage stability during normal operating conditions.

Acknowledgements. The authors wish to express their gratitude to Marco Didò of the European Commission's Joint Research Centre for computational and graphics support. They also thank the anonymous referees for their constructive comments and suggestions which have contributed to improving this manuscript. The editor thanks two anonymous referees for their assistance in evaluating this paper.

\section{References}

Albertson VD, Kappenman JG, Mohan N, Skarbakka GA. 1981. Load-Flow studies in the presence of geomagnetically-induced currents. IEEE Trans Power Apparatus Syst PAS-100: 594-607.

Bernabeu EE. 2013. Modelling geomagnetically induced currents in dominion virginia power using extreme 100 year geoelectric field scenarios - part I. IEEE Trans Power Deliv 28: 512-523.

Bolduc L. 2002. GIC observations and studies in the Hydro-Québec power system. J Atmospheric Sol-terr Phys 64: 1793-1802.

Boteler DH, Space weather effects on power systems, Space Weather Geophysical Monograph, American Geophysical Union, Washington, D. C.,Vol. 125: pp. 347-352, 2001. 
Boteler DH. 2014. Methodology for simulation of geomagnetically induced currents in power systems. J Space Weather Space Clim 4: A21.

Dong X, Study of power transformer abnormalities and IT applications in power systems, $\mathrm{PhD}$ Thesis, Virginia Polytechnic Institutem, pp. 63-87, 2002.

Dong X, Liu Y, Kappenman JG. 2001. Comparative analysis of exciting current harmonics and reactive power consumption from GIC saturated transformers. In: Proceedings of IEEE 2001 Winter Meeting. Columbus, OH, pp. 318-322.

Elovaara J. 2005. Finnish experiences on grid effects of GICs, 2nd European Space Weather Week, ESA-ESTEC, Noordwijk, The Netherlands, 14-18 November http://swe.ssa.esa.int/TECEES/ spweather/workshops/eswwII/esww2-proceedings.html\#5

Elovaara J, Finnish experience with grid effects of GICs, Space Weather - research towards Applications in Europe, Lilenstein L ed., Springer, Dordrecht, The Netherlands, pp. 311-326, 2007.

Erinmez IA, Majithia S, Rogers C, Yasuhiro T, Ogawa S, Swahn H, Kappenman JG. 2002. Application of modelling techniques to assess geomagnetically induced current risks on the NGC transmission system, paper presented at Int Counc On Large Electr Fields. Paris.

Gérin-Lajoie L, Mahseredjan J, Guillon S, Saad O. 2013. Impact of transformer saturation from GIC on power system voltage regulation, International Conference on Power Systems Transients (IPST2013). Vancouver, Canada, July 18-20 http://www.ipstconf. org/papers/Proc_IPST2013/13IPST054.pdf

Gérin-Lajoie L, Mahseredjan J, Guillon S, Saad O. 2014. Simulation of voltage collapse caused by GMDs - Problems and Solutions. Cigre, pp. 1-7.

Girgis R, Vedante K, Gramm K. Effect of geomagnetically induced currents on power transformers and power systems. Technical report, Cigre, Paris, 2012.

JASON, Impact of severe space weather on the electric grid, JSR11-320, The MITRE Corporation, 2011.

Lehtinen M, Pirjola R. 1985. Currents produced in earthed conductor networks by geomagnetically induced electric fields. Am Gheophys 3: 479-484.
NERC, GMDTF Interim Report: Effects of Geomagnetic disturbances on the Bulk power system, North American Electric Reliability Corporation, 2012.

Overbye T, Hutchins T, Shetye K, Weber J, Dahman S. 2012. Integration of geomagnetic disturbance modeling into the power flow: a methodology for large-scale system studies. North American Power Symposium (NAPS).

Pagani GA, Aiello M. 2013. The Power Grid as a complex network: a survey. Physica A 392: 2688-2700.

Piccinelli R, Krausmann E. Space weather impact on the Scandinavian Interconnected Power transmission System, EUR 27571EN, IBN 978-92-79-53761-5, 2015.

Pirjola R. 1983. Induction in power transmission lines during geomagnetic disturbances. Space Sci Rev 35: 185-193.

Pirjola R. 2008a. Study of effects of changes of earthing resistances on GICs in an electric power transmission system. Radio Sci 43: R S1004.

Pirjola R. 2008b. Effects of interactions between stations on the calculation of geomagnetically induced currents in an electric power transmission systems. Earth Planets Space 60: 743-751.

Pirjola R, Pulkkinen A, Viljanen A. 2003. Studies of space weather effects on the Finnish natural gas pipeline and on the Finnish highvoltage power system. Adv Space Res 31: 795-805.

Pulkkinen A, Bernabeu E, Eichner J, Beggan C, Thompson AWP. 2012. Generation of 100-year geomagnetically induced current scenarios. Space Weather 10: S04003.

Pulkkinen A, Pirjola R, Viljanen A. 2008. Statistics of extreme geomagnetically induced current events. Space Weather 6: S07001.

Vilianen A, Pirjola R, Wik M, Ádám A, Prácser E, Sakharov Y, Katkalov J. 2012. Continental scale modelling of geomagnetically induced currents. J Space Weather Space Clim 2: A17.

Walling RA, Khan AH. 1991. Characteristics of transformer excitingcurrent during geomagnetic disturbances. IEEE Trans Power Deliv 6: $1707-1714$.

Wik M, Viljanen A, Pirjola R, Pulkkinen A, Wintoft P, Lundstedt H. 2008. Calculation of geomagnetically induced currents in the $400 \mathrm{kV}$ power grid in southern Sweden. Space weather 6: S07005.

Cite this article as: Piccinelli R, Krausmann E. 2018. North Europe power transmission system vulnerability during extreme space weather. J. Space Weather Space Clim. 8: A03 